\section{Assessment and support of children and adolescents with gender dysphoria}

\author{
Gary Butler, ${ }^{1,2,3}$ Nastasja De Graaf, ${ }^{3}$ Bernadette Wren, ${ }^{3}$ \\ Polly Carmichael ${ }^{3}$
}

\section{INTRODUCTION}

There has been a large increase in the number of children and adolescents who question conventional gender expectations and seek recognition and acceptance of their gender diversity, wishing to develop a body that is congruent with their gender feelings. ${ }^{1}$ Professionals may be unsure how best to provide supportive care, how to access the national Gender Identity Development Service (GIDS) for children and adolescents, or how to deal with a transgender young person presenting with another clinical problem unrelated to their gender transition. Faced with very distressed young people, they may feel under pressure to initiate physical intervention without consultation with psychosocial colleagues. It is important that all professionals are aware of the care pathway for transgender children that may be of relevance in a range of paediatric settings. The purpose of this practice review is to present an up-to-date perspective on the care of transgender children and adolescents to guide management and to enable the provision of a practical, evidence-based approach to their support.

\section{FREQUENCY OF GENDER QUESTIONING IN CHILDREN}

Gender atypical behaviour is common among young children and can be part of general development. It is difficult to determine the exact incidence and prevalence of more intense and long-standing gender dysphoria (GD) in the UK and elsewhere as the total number of children and young people referred to the GIDS has risen exponentially since $2011^{2}$ (figure 1 ). A striking feature of this increase is the large proportion of birth-registered females from 2011 onwards. This increase

\footnotetext{
${ }^{1}$ Department of Paediatric and Adolescent Endocrinology, University College London Hospital NHS Trust, London, UK

${ }^{2} U C L$ Great Ormond Street Institute of Child Health, London, UK

${ }^{3}$ National Gender Identity Development Service,

Tavistock and Portman NHS Trust, London, UK
}

Correspondence to Professor Gary Butler, Department of Paediatric and Adolescent Endocrinology, University College London Hospital NHS Trust, London NW1 2PQ, UK; gary.butler@ucl.ac.uk and the change in sex ratio is also seen in other countries. ${ }^{1}$ The reasons are not fully explicable and a number of questions arise. Is this increase due mostly to the greater tolerance of gender-diverse expression in westernised society? Is male status still regarded as preferable? Are all referrals to a specialist service appropriate and do all these young people have ascertainable GD? What are the benefits, as well as the possible harms, in supporting and helping these young people at different stages of development?

\section{THE GIDS}

Support for children and adolescents up to the age of 18 years has been provided through the Tavistock and Portman NHS Trust in London for over 20 years. The GIDS was nationally commissioned by NHS England in 2009 and extended to Leeds in 2012, providing regular outreach clinics in other areas of the UK. Endocrine evaluation and support has been provided through University College Hospital London for over two decades, and Leeds Children's Hospital since 2013. Care is provided according to an agreed service schedule, ${ }^{3}$ taking into account international guidance from the World Professional Association for Transgender Health (WPATH) ${ }^{4}$ and the recent guidelines from the Endocrine Society. ${ }^{5}$

Eirth-assigned males $\quad$ Birth-assigned females

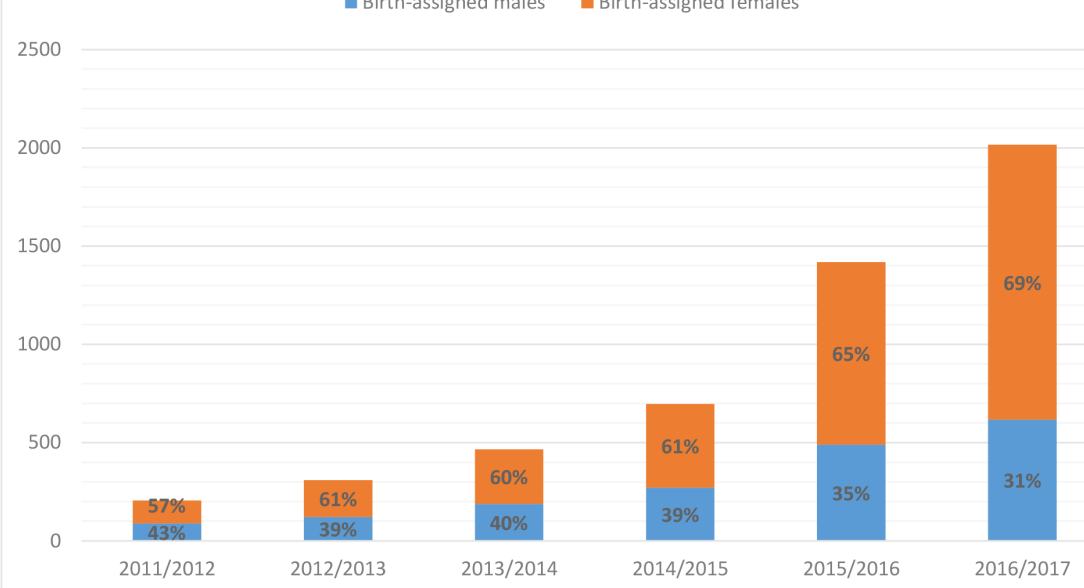

Figure 1 Referrals to the Gender Identity Development Service by birth-registered gender, April 2011-April 2017. at least 6 months (box 2).

\section{RANGE OF GENDER VARIANT PRESENTATION TO THE GIDS}

CRITERIA FOR THE DIAGNOSIS OF GD

The Diagnostic and Statistical Manual of Mental Disorders, Fifth Edition ${ }^{6}$ provides one overarching diagnosis of GD with separate specific criteria for children and for adolescents and adults. The diagnosis involves a difference between one's experienced/expressed gender and assigned gender, and significant distress or problems in everyday functioning. Diagnosis can be made when at least two features have been present for 6 months or more (box 1). For children, cross-gender behaviours may start between the ages 2 and 4 , the same age at which most typically developing children begin showing gendered behaviours and interests. The diagnosis of GD involves at least six of the criteria and an associated significant distress or impairment in function, lasting

Young people referred to the GIDS are a heterogeneous group and may identify in various ways. Most young people coming to gender services report feeling more in line with the opposite gender than that in which they were registered at birth (box 3). This may be accompanied by feelings of distress, particularly around the body which does not fit with the experienced gender identity. Young people who do not identify within the two-binary model of gender, but rather hold a gender identity other than male or female, are referred to as non-binary. ${ }^{7}$ Around $11 \%$ of the referred adolescents report a non-binary identity and often use labels such as 'gender neutral' or 'gender fluid' (Twist and De Graaf, submitted, 2017). Some young people 
Box 1 Criteria for the diagnosis of gender dysphoria in adults

- A marked incongruence between one's experienced/expressed gender and primary and/or secondary sex characteristics.

- A strong desire to be rid of one's primary and/or secondary sex characteristics.

- A strong desire for the primary and/ or secondary sex characteristics of the other gender.

- A strong desire to be of the other gender.

- A strong desire to be treated as the other gender.

- A strong conviction that one has the typical feelings and reactions of the other gender.

are unsure about their gender identity and seek support from professionals to help them reflect and explore. Although gender-diverse young people tend to experience some psychological or social difficulties, gender diversity is not necessarily associated with distress. ${ }^{4}$

\section{APPROPRIATE ASSESSMENTS PRIOR TO REFERRAL}

The GIDS is an unusual multidisciplinary service in that the initial assessment phase

Box 2 Criteria for the diagnosis of gender dysphoria in children and adolescents

- A strong desire to be of the other gender or an insistence that one is the other gender.

- A strong preference for wearing clothes typical of the opposite gender.

- A strong preference for cross-gender roles in make-believe play or fantasy play.

- A strong preference for the toys, games or activities stereotypically used or engaged in by the other gender.

- A strong preference for playmates of the other gender.

- A strong rejection of toys, games and activities typical of one's assigned gender.

- A strong dislike of one's sexual anatomy.

- A strong desire for the physical sex characteristics that match one's experienced gender.
Box 3 A glossary of useful

terminology

\section{Gender identity}

- Gender identity is the individual's deeply held personal sense of their own gender as male or female, neither or both.

\section{Gender dysphoria}

- Gender dysphoria, as a general descriptive term, refers to an individual's discontent with their 'assigned' gender and their identification with a gender other than that associated with their birth sex based on physical sex characteristics. 'Dysphoria' relates to the distress and unease experienced. The term is more specifically defined when used as a diagnosis (Diagnostic and Statistical Manual of Mental Disorders, Fifth Edition).

\section{Transgender}

- Transgender refers to the broad spectrum of individuals who identify with a gender other than that associated with their birth sex.

Gender variance and gender diversity

- Gender variance and gender diversity are umbrella terms used to describe the wide range of gender identifications outside conventional gender categories.

\section{Non-binary}

- Non-binary is a lack of identification with conventional maleness or femaleness. Non-binary people may express features of both genders or neither.

\section{Transman/transboy}

- A person born phenotypically female (natal female), registered (assigned) female at birth, who identifies as male. Also known as female to male.

\section{Transwoman/transgirl}

- A person born phenotypically male (natal male), registered (assigned) male at birth, who identifies as female. Also known as male to female.

\section{GnRH analogue}

- GnRH analogue known colloquially as 'the blocker'. A longer acting version of the naturally occurring gonadotropin-releasing hormones (GnRH) such as triptorelin (Gonapeptyl Depot or Decapeptyl SR) used to prevent pituitary gonadotropin (follicle stimulating

Continued

\section{Box 3 Continued}

hormone FSH and luteinising hormone LH) secretion by competitive inhibition of the $\mathrm{GnRH}$ receptor.

\section{Cross-sex hormones}

- Cross-sex hormones (also known as gender-affirming hormones): physiological doses of testosterone in transboys and oestradiol in transgirls used to induce secondary sex changes associated with the gender of identification.

by the psychosocial team precedes medical involvement, and is aimed at understanding the young person's development and gender identification in the context of their family background and life experiences. $^{8}$ It takes account of how they feel about their gender and their body now and in the past, the context in which the gender dysphoric feelings arose and intensified, how these feelings are being managed in everyday life, and what hopes are held for proceeding in the future. A range of psychometric measures are used to assess behavioural and emotional functioning, including features of autistic spectrum disorder and self-harm. Around 35\% of referred young people present with moderate to severe autistic traits. The psychosocial assessment also explores the child's expectations of what will change if a social or physical transition is sought, as well as their understanding of the losses that may be incurred. With the adolescents, there is an indepth consideration of their sexuality and fertility, and possible preservation approaches are discussed. The attitude of important people in the child's life towards GD needs to be explored and understood. The child's mood and anxiety levels are evaluated. If there are concerns about self-harm or other kinds of risk, the local child and adolescent mental health service will be asked to provide further psychiatric and multidisciplinary input as appropriate.

The assessment period usually takes 6 months or more over a minimum of four to six sessions. At the end of the assessment, it may be possible to confirm or exclude a diagnosis of GD. Further treatment decisions are discussed with the family and agreed by the wider multidisciplinary team. One outcome may be to continue exploration of gender feelings and careful consideration of different developmental pathways and identity outcomes. A referral may also be made to the paediatric endocrinology 
300

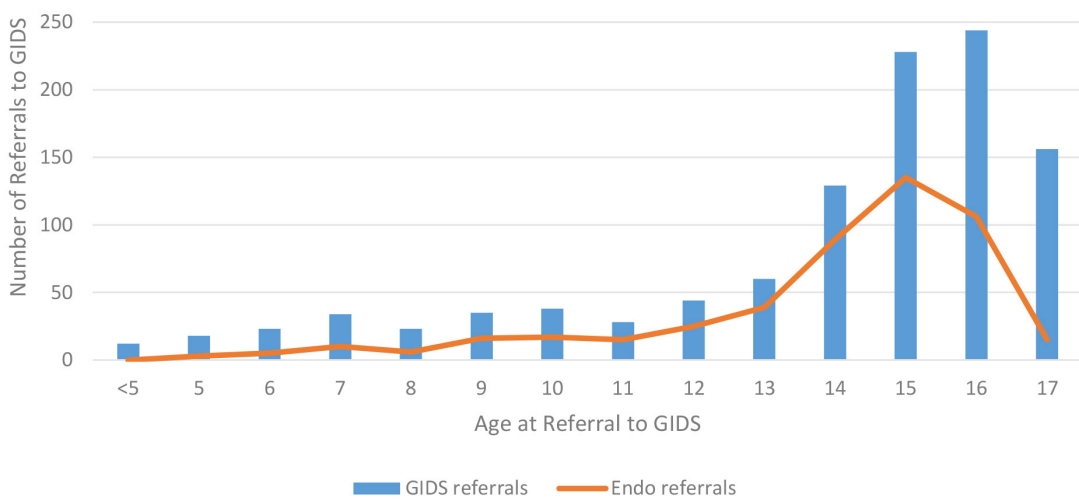

Figure 2 Numbers of young people presenting to the Gender Identity Development Service (GIDS) between 2010 and 2013 by age at initial referral (blue bars) and the proportion who had been referred on to the paediatric endocrinology clinics by 2017 (orange line).

team for consideration of puberty suspension with a gonadotropin-releasing hormone analogue (GnRHa) when the young person fulfils the minimum eligibility criteria, such as Tanner puberty stage 2.459 Once the paediatric team becomes involved, the child and family still continue regular sessions with their mental health clinician, and the two arms of the service work closely together. Following psychosocial assessment at the GIDS, on average 38\%-40\% of all clients attend the joint endocrine clinics, although this varies across the age range. Figure 2 highlights the age at which young people were referred to the GIDS, not the age at which they were referred to endocrinology (at a mean age of 14.4 years). The percentage referred at age 16 or 17 years is lower as many transition then to adult services to access physical treatment directly.

\section{ASSESSMENTS IN PAEDIATRIC CARE}

What is the correct approach when a young person questioning their gender is referred to the GIDS child and adolescent medical team? The increasing number of referrals and systematic data collection carried out since the GIDS was nationally commissioned in 2009 has allowed us to make some evidence-based evaluations of this process. Our current best practice is described in what follows. Even though the initial presentation of GD may be through paediatric services, a specialist psychological assessment should be requested before any physical treatment can be considered as the intervention itself can mask the adequacy of the evaluation.

GIDS, Gender Identity Development Service.

\section{Pubertal status} crine Society and WPATH guidelines and UK service specifications, that a young person has commenced puberty before any intervention is agreed, as the GD may resolve once puberty commences. ${ }^{45}$ Figure 2 demonstrates the low absolute numbers and proportion of prepubertal children with persistent GD. Hence a clinical examination for pubertal staging is required unless confirmed as in the completing phase (eg, postmenarche from history). The majority of referrals to the GIDS and when referred on to the joint endocrine clinics in Leeds and London are postpubertal, two-thirds being over 15 years of age.

\section{Karyotype}

Initially the approach with GD was similar to that for disorder of sex development, with a karyotype being routinely requested. An audit of UK clinics from 2013 to 2015 (Table 1) revealed no differences from cytogenetic surveys of the UK newborn population and elsewhere. ${ }^{10} 11$ Therefore, routine karyotyping of a child or adolescent with GD is not required
It is a requirement, according to the Endo-

unless any specific clinical features determine this to be necessary.

\section{Radiological assessments}

Other diagnostic procedures employed in DSD evaluations were also routine in the GD clinic until recently. Transabdominal pelvic ultrasound scans were carried out in all phenotypic females, but between 2009 and 2013 only one congenital anomaly was found. Polycystic or multicystic ovaries were commonly seen, not necessarily associated with clinical or biochemical hyperandrogenism. Pelvic ultrasonography is therefore not required.

A bone age is routinely requested in a young person who has not completed puberty as it may be helpful for height prediction. The effect on adult height of the manipulation of the adolescent growth spurt using GnRHa and the induction of a second puberty with exogenous cross-sex hormones is not yet fully elucidated.

Bone densitometry is performed at the initial assessment and then annually until more information is known. The longer term effect of ablating sex hormone secretion is still under review, and preliminary reports from the Netherlands suggest that bone mineral content lost during treatment may not be fully recovered, although this may be dependent on the age at start of treatment. The longer term health consequences are unclear. ${ }^{12} 13$

\section{Endocrine assessments}

Endocrine assessment of the reproductive axis of the birth-registered phenotypic male does not usually demonstrate any anomalies, sex hormone and gonadotropin concentrations merely reflecting the stage of puberty attained. A low testosterone may result from self-medication with antiandrogens such as spironolactone to alleviate the distress of progressive virilisation. Exogenously administered oestrogen may be detectable as an abnormal oestradiol:testosterone ratio in the serum, and on examination breast development may be present and the testes may be smaller than expected for the pubertal stage.

Table 1 Karyotypes performed in young people attending GIDS England and Wales, and from Scotland and Northern Ireland (2009-2015), and frequencies of aneuploidy ${ }^{10}$

\begin{tabular}{|c|c|c|c|}
\hline $46, X X$ & 269 & & \\
\hline $46, X Y$ & 177 & & \\
\hline Total & 446 & & \\
\hline $\begin{array}{l}\text { New sex chromosome } \\
\text { aneuploidies }\end{array}$ & $47, X Y Y$ & GIDS: 1 in 446 & Population: 1 in $426-523$ \\
\hline $\begin{array}{l}\text { New autosomal } \\
\text { aneuploidies }\end{array}$ & $\begin{array}{l}46 X X, t(7 ; 13)(p 21 ; q 31) m o s \\
47, X X,+\operatorname{mar}[10] / 46, X X[20]\end{array}$ & GIDS: 1 in 223 & Population: 1 in 138-164 \\
\hline
\end{tabular}


A search for sources of hyperandrogenism in adolescent birth-registered phenotypic females in an attempt to explain the dysphoria has not elucidated any differences from a control population selected from females of similar ages within the same department undergoing evaluation of the reproductive and adrenal axes. Neither basal nor stimulated adrenal androgens or testosterone was different between the GD or control populations. It therefore appears that GD is not associated with excess androgen production in postpubertal phenotypic females. Use of contraceptive pill/oral contraceptives to halt menstruation will show the expected hormone suppression.

\section{Fertility preservation}

The initiation of GnRHa therapy halts gonadotropin and gonadal sex hormone secretion, but also suspends gonocyte maturation. Oocytes remain dormant and spermatogenesis is halted. Young people and their families are routinely counselled about fertility loss resulting from the physical treatment. Various medical options are considered in case of a desire to revert to their birth gender in subsequent years after irreversible treatments have occurred, or in case assisted conception techniques might be called on in adult life to assist with starting a family. A referral to specialist fertility services is always offered in addition. ${ }^{14} 15$

Phenotypic males are counselled about semen harvesting, which is possible once puberty is under way. By Tanner stage 3 ejaculation can take place. Even if the sperm count is low at that stage, there may be sufficient for preservation for future assisted conception. The dysphoria however may be too extreme to consider semen collection by masturbation. Electroejaculation may then be helpful.

The situation is more complex in phenotypic females. Prior to menarche, ovarian biopsy is possible but not routinely available at present. Mature oocyte harvesting post menarche requires the maturity to engage with gonadotropin pretreatment and transvaginal extraction. The access to reproductive services for transgender people is very patchy at present, and regional National Health Service (NHS) funding is not always available.

Some young people, whose primary objective is to halt the pubertal progress, will choose to postpone the fertility preservation decision until older. If they want to preserve fertility after having started $\mathrm{GnRHa}$, it may take 6 months or more for the reproductive axis to recover, and
Box 4 Useful first-line

investigations in a child or

adolescent presenting with gender dysphoria

Full blood count.

- Iron/ferritin.

- U\&E, LFT.Renal and liver function tests

- Bone profile.

- Vitamin D.

- Testosterone.

- Oestradiol.

- Follicle stimulating hormone FSH and luteinising hormoneLH.

- Prolactin.

- Bone age in premenarchal female to male or prepuberty/inpuberty male to female.

- Bone density scan.

the reproductive capacity will only be the same as at the point of starting blocker treatment. The need to stop the GnRHa in itself may be a barrier to pursuing gamete harvesting for some young people as endogenous sex hormone effects will return, although temporarily.

\section{Recommended approach to initial investigations and monitoring of treatments}

Investigations are purely required to evaluate adolescent health status and to measure baseline spontaneous gonadotropin and sex hormone production, as our search for genetic, endocrine and radiological diagnostic markers of a dysphoria-related state has proven negative. As reduction of sex hormone production in mid-adolescence to late adolescence may well have effects on reducing energy and psychological well-being, parameters such as full blood count, iron, vitamin D and thyroid status are important to ascertain (box 4). Bone mineralisation may be reduced on account of the GnRHa intervention; thus, bone density evaluation is currently recommended. Furthermore, since the effect of the blockade of sex hormones on the intensity, duration and outcome of the adolescent developmental process is still largely unknown, height, weight and body proportion estimation should be measured with regular bone age estimations.

\section{PRIMARY INTERVENTION: GnRHa}

In those whose GD is clearly established and long-standing and who have participated fully in the multidisciplinary assessment process at the GIDS, GnRHa is the recommended first-stage medical intervention. This provides a physically reversible option to suspend sex hormone production, reduce the anxiety of ongoing physical development and allow further space for psychotherapeutic intervention and exploration.

This treatment is also requested in early pubertal children as a way to prevent the development of those unwanted bodily characteristics that may make 'passing' in the preferred sex difficult in later life (deep voice, facial hair and changes, breasts and so on) should this be their wish, and to render unnecessary certain surgical interventions such as mastectomy.

For children under 16, parents have the responsibility to agree to support any treatment (or not), with the clinicians determining the child's capacity to give consent, upholding the child's best interests in line with the UK General Medical Council guidance. ${ }^{16}$ Young people over 16 are deemed as having the capacity to give their own consent to treatment. For all young people there is a requirement that they are helped to develop an adequate understanding of what is to be offered-both its risks and benefits, and the alternatives to treatment. An age-related appreciation of the potential loss of biological fertility is also a prerequisite. In practice adolescents vary greatly in their level of maturity, and it is important, when clinicians have any concern about the capacity to consent, that the parent/ carer be fully engaged to participate in the decision. If the parents or carers object to treatment, this poses a complex ethical and social challenge.

According to the WPATH and the recently updated Endocrine Society guidelines, GnRHa may be offered once an adolescent has demonstrated the first signs of physical puberty (Tanner stage 2 in either sex). ${ }^{45}$ This internationally endorsed approach stems from the fact that between $73 \%$ and $88 \%$ of children attending gender identity clinics who present prepubertally, whether they have socially transitioned or not, are likely not to continue with their intention of changing their gender once puberty has started. ${ }^{17}$ Some may 'desist' completely, with their initially perceived dysphoric feelings pointing towards developing homosexuality, while others may wish to pursue an expression of their gender identity which does not require medical intervention.

$\mathrm{GnRHa}$ is prescribed in standard doses for a minimum of a year before additional medical interventions can be considered. This is to allow further psychotherapeutic assessment to take place once the anxiety 
and distress related to the progression in puberty and processes such as menstruation, erections and nocturnal emissions are reduced or stopped. All young people are counselled that GnRHa cannot be considered as a contraceptive, and that additional anticonceptive methods should be used.

\section{Psychological benefits of GnRHa treatment}

Puberty suspension with GnRHa constitutes a treatment in its own right, meaning that it is not indicated purely as a preliminary stage before the next stage of treatment towards gender reassignment, namely gender-affirming cross-sex hormones. ${ }^{918}$ This puts a responsibility on the parents/carers, as well as clinicians in multidisciplinary teams, to try and ensure that time and opportunity are made for thoughtful and well-informed considerations of future options.

Most young people welcome the 'blocker' intervention as helping them to feel more comfortable in their bodies while holding future gender options open. However, our psychometric data suggest that although it permits further time for exploration, the impact on reducing psychological difficulties is limited. Further research is needed to determine whether GnRHa treatment is beneficial for young people of all ages, or whether some age groups may benefit more from this particular treatment than others, perhaps younger rather than older adolescents?

\section{ELIGIBILITY FOR CROSS-SEX HORMONE/GENDER-AFFIRMING HORMONE TREATMENT}

Cross-sex hormone treatment, currently referred to as gender-affirming hormone treatment (namely the induction of the opposite-biological sex puberty using testosterone in a phenotypic female and oestradiol in a phenotypic male), may be considered once full commitment to the preferred gender has been confirmed, and further detailed psychological exploration of the gender identity has been conducted. At the GIDS there are psychosocial criteria that need to be met, including that the young person shows some evidence of presenting coherently in the gender role with which they identify and that mental health difficulties are not escalating. Smoking and vaping are actively discouraged.

The capacity to give informed consent is an absolute requirement prior to commencing sex hormone treatment, ideally with the support of the parents, although after 16 years of age the latter is not needed under the English, Welsh and Scottish law. It is acknowledged by the Endocrine Society that a young person under the age of 16 may not have sufficient psychological maturity to commit to such a lifelong and life-changing decision, ${ }^{5}$ and no evidence is available to understand the effects of giving gender-affirming hormone treatment before 16 years. ${ }^{4} \mathrm{~A}$ systematic review of the available evidence has been published recently by NHS England Clinical Commissioning Policy. ${ }^{19}$

\section{Regimens for gender-affirming sex hormone treatment}

Therapeutic regimens suggested for gender-affirming hormone treatment with testosterone or oestradiol reflect the process of the induction of puberty that is traditionally done in paediatric endocrine practice to ensure maximum benefit from the gradual introduction to sex hormones. ${ }^{520}$ The dose increase schedule is conducted more slowly in those who have never completed puberty in their birth sex to allow emotional and social maturation, as well as the completion of the adolescent growth spurt in height. Although physical changes are the goal from the young person's perspective, a gain in confidence is the most important marker of the success of treatment. Alterations in mood, including the development of irritability and anxiety, are risks of this treatment and are dose-dependent. However, despite the development of mood variations and anxiety, very few young people request to stop treatment at this stage. ${ }^{21}$ Fifty-five per cent of those seen initially at the GIDS and over 90\% of those attending endocrinology clinics request further support from adult gender identity clinics. GnRHa is continued during feminisation in transgirls as it is difficult to suppress spontaneous testosterone production fully with exogenous oestrogen. It is usual to continue this dual therapy until gonadectomy or an alternative long-term solution is decided on. In transboys, once testosterone concentrations are elevated into the adult male range, GnRHa can be stopped as the recurrence of menses then is unlikely.

\section{FURTHER SUPPORTIVE TREATMENTS}

The GIDS is commissioned to look after young people up to the age of 18 years, after which referral may be offered by one of the currently nationally commissioned UK adult gender identity clinics. There, after further assessment, the treatment schedule will usually be reauthorised and the young person may at their own request be referred for surgical interventions. This can include mastectomy, frequently requested by transboys, and gender affirmation surgery less often. Male-to-female feminising genitoplasty is a well-established procedure where the penoscrotal skin is refashioned to produce the vagina and labia with preservation of the glans and neurovascular bundle to form a clitoris capable of orgasm. Concern now exists that the early blockade of puberty may result in insufficient genital skin being available for this procedure.

Female-to-male surgery is more complex. Options include metoidioplasty, where the testosterone-enhanced clitoris is tubularised and a scrotum is constructed from the labia. In full male genital reconstruction, a penis can be formed from either abdominal, thigh or forearm skin flaps. A hydraulic implant is inserted in a newly fashioned scrotum to provide controllable erectile function. This approach requires several surgical procedures and leaves extensive scarring.

\section{LONG-TERM OUTCOMES OF GD MANAGEMENT}

In the UK, as yet there is little evidence of the longitudinal outcome from the GIDS. One published study showed that psychological support and puberty suppression were both associated with an improved global psychosocial functioning in adolescents with GD.22 One clear difficulty in tracking people is the frequent change in nominal and legal identity, including NHS number in those referred on to adult services and thus to date they have not been able to be followed up. The few longitudinal follow-up studies that have been published in the Netherlands have generally shown a high satisfaction outcome, particularly from those who have gone through surgical affirmation of their identified gender. ${ }^{23}{ }^{24}$ These reported outcomes however are only from a highly selected group of fully dysphoric, well-adjusted individuals with few comorbidities and may not represent the full spectrum of gender diversity. Much further work needs to be done in this area.

\section{ALTERNATIVE TREATMENTS WHICH CAN BE OFFERED TO A GENDER VARIANT OR NON-BINARY GENDER- IDENTIFYING PERSON}

GD has typically been associated with a full identification with the opposite gender, and until recently a full social and legal transition was a requirement before receiving gender-affirming hormones. However, a significant number of young people are unsure of their eventual gender status or may retain feelings related to 
their birth sex/gender as well as to their preferred gender, that is, a mixture of both. Non-binary gender identification is now increasingly encountered in clinical settings.

In some situations, simple management of the anxiety generated by birth-sex features in transboys such as menstruation with commonly used gynaecological treatments is a good solution. The combined contraceptive pill/oral contraceptives taken continuously with a break one to two times a year for a menstrual bleed, or a continuous progestogen such as norethisterone, may be prescribed. Depot progesterone implants, either subcutaneously or intrauterine, have variable success in halting menstruation. In young transgirls, alternative antiandrogen therapies such as spironolactone or cyproterone acetate are not recommended due to the high incidence of side effects and low efficacy, but topical treatments such as eflornithine cream (Vaniqa) may help to reduce unwanted facial and body hair growth.

\section{CONCLUSION}

The UK, along with other developed countries, is seeing an increase in the number of young people who are questioning their gender and taking the decision to pursue social and medical transition in line with their identified gender which may be non-binary. While professionals need to treat young people with respect and to acknowledge the intensity of their gender identification, much is still to be ascertained about the impact of medical intervention. The best practice in this field currently involves close collaborative working in multidisciplinary teams.

Acknowledgements The authors thank all the staff of the GIDS.

Collaborators Kirpal Adu-Gyamfi, Caroline Brain, Claire Goedhart, Sara Kleczewski, Elaine Perkins, Tiffani Rees, Alice Roberts and Russell Viner from University College London NHS Trust; Sabah Alvi, Bindu Avatpalle, Paul Carruthers, Talat Mushtag and Jenny Walker from Leeds Children's Hospital, Leeds Teaching Hospitals NHS Trust; and Noina Abid from Belfast's Royal Hospital for Sick Children and Guftar Shaikh from Royal Hospital for Sick Children, Glasgow for also contributing karyotype results.
Contributors GB conceived the idea, analysed the biological data and wrote the paediatric medical sections. NDG analysed the demographic and psychological data and cowrote those sections with BW. PC has had significant input to the final document.

Competing interests None declared.

Provenance and peer review Commissioned; internally peer reviewed.

(C) Article author(s) (or their employer(s) unless otherwise stated in the text of the article) 2018. All rights reserved. No commercial use is permitted unless otherwise expressly granted.

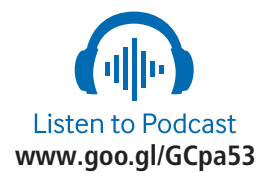

$$
\text { A) Check for updates }
$$

To cite Butler G, De Graaf N, Wren B, et al. Arch Dis Child 2018;103:631-636.

Received 16 February 2018

Revised 13 March 2018

Accepted 18 March 2018

Published Online First 12 April 2018

Arch Dis Child 2018;103:631-636.

doi:10.1136/archdischild-2018-314992

\section{REFERENCES}

1 Aitken M, Steensma TD, Blanchard R, et al. Evidence for an altered sex ratio in clinic-referred adolescents with gender dysphoria. J Sex Med 2015;12:756-63.

2 Zucker KJ, Lawrence AA. Epidemiology of gender identity disorder: recommendations for the standards of care of the world professional association for transgender health. Int J Transgend 2009;11:8-18.

3 NHS England. NHS standard contract for gender identity development service for children and adolescents. https://www.england.nhs.uk/wp-content/ uploads/2017/04/gender-development-servicechildren-adolescents.pdf

4 Coleman E, Bockting W, Botzer M, et al. Standards of care for the health of transsexual, transgender, and gender-nonconforming people, version 7. Int J Transgend 2012;13:165-232.

5 Hembree WC, Cohen-Kettenis PT, Gooren L, et al. Endocrine treatment of gender-dysphoric/ gender-incongruent persons: an endocrine society* clinical practice guideline. J Clin Endocrinol Metab 2017;102:3869-903.

6 American Psychiatric Association. Diagnostic and statistical manual of mental disorders (DSM-5). 2013.

7 Richards C, Bouman WP, Seal L, et al. Non-binary or genderqueer genders. Internat Rev Psych 2016:28:95-102.

8 Di Ceglie D. Gender identity disorder in young people. Adv Psych Treatment 2000;6:458-66.
9 Delemarre-van de Waal HA, Cohen-Kettenis PT. Clinical management of gender identity disorder in adolescents: a protocol on psychological and paediatric endocrinology aspects. Eur J Endocrinol 2006;155S:131-S137.

10 Jacobs PA, Browne C, Gregson N, et al. Estimates of the frequency of chromosome abnormalities detectable in unselected newborns using moderate levels of banding. J Med Genet 1992;29:103-8.

11 Nielsen J, Wohlert M. Sex chromosome abnormalities found among 34,910 newborn children: results from a 13-year incidence study in Arhus, Denmark. Birth Defects Orig Artic Ser 1990;26:209-23.

12 Klink D, Caris M, Heijboer A, et al. Bone mass in young adulthood following gonadotropin-releasing hormone analog treatment and cross-sex hormone treatment in adolescents with gender dysphoria. J Clin Endocrinol Metab 2015;100:E270-75.

13 Vlot MC, Klink DT, den Heijer M, et al. Effect of pubertal suppression and cross-sex hormone therapy on bone turnover markers and bone mineral apparent density (BMAD) in transgender adolescents. Bone 2017:95:11-19.

14 Finlayson C, Johnson EK, Chen D, et al. Proceedings of the working group session on fertility preservation for individuals with gender and sex diversity. Transgend Health 2016;1:99-107.

15 Nahata L, Tishelman AC, Caltabellotta NM, et al. Low fertility preservation utilization among transgender youth. J Adolesc Health 2017;61:40-4.

16 The duties of a doctor registered withthe General Medical Council. https://www.gmc-uk.org/0_18_ years_English_1015.pdf_48903188.pdf

17 Wallien MS, Cohen-Kettenis PT. Psychosexual outcome of gender-dysphoric children. J Am Acad Child Adolesc Psychiatry 2008;47:1413-23.

18 Cohen-Kettenis PT, Steensma TD, de Vries AL. Treatment of adolescents with gender dysphoria in the Netherlands. Child Adol Psych Clinics 2011;20:689-700.

19 Clinical commissioning policy: prescribing of cross-sex hormones as part of the gender identity development service for children and adolescents. https://www. england.nhs.uk/commissioning/wp-content/uploads/ sites/12/2016/08/clinical-com-pol-16046p.pdf

20 de Vries AL, Cohen-Kettenis PT. Clinical management of gender dysphoria in children and adolescents: the Dutch approach. J Homosex 2012;59:301-20.

21 Vrouenraets LJ, Fredriks AM, Hannema SE, et al. Perceptions of Sex, Gender, and Puberty Suppression: A Qualitative Analysis of Transgender Youth. Arch Sex Behav 2016:45:1697-703.

22 Costa R, Dunsford M, Skagerberg E, et al. Psychological support, puberty suppression, and psychosocial functioning in adolescents with gender dysphoria. J Sex Med 2015;12:2206-14.

23 de Vries AL, Steensma TD, Doreleijers TA, et al. Puberty suppression in adolescents with gender identity disorder: a prospective follow-up study. J Sex Med 2011;8:2276-83.

24 de Vries AL, McGuire JK, Steensma TD, et al. Young adult psychological outcome after puberty suppression and gender reassignment. Pediatrics 2014;134:696-704. 